\title{
Minimum energy control of passive tracers advection in point vortices flow
}

\author{
Carlos Balsa ${ }^{1}$, Olivier Cots $^{2}$, Joseph Gergaud ${ }^{2}$, and Boris Wembe ${ }^{2}$ \\ 1 Research Centre in Digitalization and Intelligent Robotics (CeDRI), Instituto \\ Politécnico de Bragança, Campus de Santa Apolónia, 5300-253 Bragança, Portugal; \\ email: balsa@ipb.pt. \\ 2 Toulouse Univ. INP-ENSEEIHT-IRIT, UMR CNRS 5505, 2 rue Camichel, 31071 \\ Toulouse, France; e-mail: (olivier.cots, joseph.gergaud, boris.wembe)@irit.fr.
}

\begin{abstract}
In this work we are interested in controlling the displacement of particles in interaction with $N$ point vortices, in a two-dimensional fluid and neglecting the viscous diffusion. We want to drive a passive particle from an initial point to a final point, both given a priori, in a given finite time, the control being due to the possibility of impulsion in any direction of the plane. For the energy cost, the candidates as minimizers are given by the normal extremals of the Pontryagin Maximum Principle (PMP). The transcription of the PMP gives us a set of nonlinear equations to solve, the so-called shooting equations. We introduce these shooting equations and present numerical computations in the cases of $N=1,2,3$ and 4 point vortices. In the integrable case $N=1$, we give complete quadratures of the normal extremals.
\end{abstract}

Keywords: Helhmoltz-Kirchhoff $N$ vortices model, Energy minimization, Pontryagin Maximum Principle, Indirect shooting method.

\section{Introduction}

This work is concerned with the control of the displacement of particles in interaction with point vortices, in a two-dimensional fluid, where the viscous diffusion is neglected, which is equivalent to using the Euler equation instead of the Navier-Stokes equation as the mathematical model of the fluid flow. We refer to Ref. [12] for details about vortex theory.

In most of the control problems, concerning realistic flows, the solution is achieved by means of simplified models such as point vortex [17]. There is a special interest in the use of control methods applied to vortex dynamics in the fields of geophysical fluid dynamics, aeronautic and hydrodynamic [16]. In the context of hydrodynamics, the fish-like locomotion and autonomous underwater vehicles are applications of point vortex that have received some attention in the last years due to the necessity of data sampling in the oceans water $[13,10,8]$. Moreover, from the dynamics of such systems there is an intense activity research initiated by Poincaré [14] to compute periodic trajectories avoiding collisions and 
such techniques lead to the concept of choregraphy developed by [6] for the Nbody problem and [5] for the $\mathrm{N}$-vortex system, showing the relations between both dynamics in the Hamiltonian frame [11]. From the control point of view, there is a lot of development related to space navigation for the N-body problem, see [3], valuable in our study for ship navigation in the N-vortex problem.

In this work we apply the Pontryagin Maximum Principle (PMP) to control the displacement of a passive particle (which is by definition a zero circulation vortex), influenced by vortex points located around it, in an optimal way (we consider the energy cost, see [2] for time minimization). This problem is a particular case of the challenging "Problem 2" included in [16]. A passive particle is small enough not to perturb the velocity field, but also large enough not to perform a Brownian motion. Particles of this type are the tracers used for flow visualization in fluid mechanics experiments [1]. We consider also that the passive particles have the same density of the fluid in which it is embedded. Explicitly we want to drive a passive particle from an initial starting point to a final terminal point, both given a priori, in a given finite time. Here, the vortex dynamics is governed by $N$ point vortices and the control is due to the possibility of impulsion in any direction of the two dimensional plane (a sufficiently long time is considered so that the optimal control remains bounded and its amplitude is small enough).

The article is structured as follows: Section 2 is devoted to the statement of the control problem. The maximum principle is stated in Section 3, with some results in the one vortex case. The cases of 2,3,4 vortices are treated in Section 4 .

\section{Vortex Dynamics and Statement of the Problem}

We give in this section a short description of the vortex dynamics and we refer to [12] for more details. This description is followed by the formulation of the control problem addressed in this work.

Let us consider the case of a two-dimensional fluid, for which the incompressible Euler equations are given by

$$
\frac{\partial \nu}{\partial t}+(\nu \cdot \nabla) \nu=-\nabla p, \quad \nabla \cdot \nu=0
$$

where $\nu$ stands for $\nu(X, t):=\left(\nu_{1}(X, t), \nu_{2}(X, t)\right)$ and represents the velocity field and $p$ is the pressure of the fluid. Due to $\nabla \cdot \nu=0$ (the incompressibility equation) from (1), one can write $\nu=\left(\nu_{1}, \nu_{2}\right)=:\left(\partial_{y} \Psi,-\partial_{x} \Psi\right)$ where $\Psi$ is called the streamfunction. Besides, let $\mathbf{w}$ denote the viscosity vector and introduce $\tilde{\nu}:=(\nu, 0)$, then $\mathbf{w}$ is given by the relation $\mathbf{w}=\nabla \wedge \tilde{\nu}=\left(0,0, \partial_{x} \nu_{2}-\partial_{y} \nu_{1}\right)=:(0,0, \omega)$, and with the two previous formulas, one can deduce the Poisson equation satisfied by $\Psi$, that is $\nabla^{2} \Psi=-\omega$. The resolution of this equation leads to

$$
\Psi(X, t)=\frac{1}{2 \pi} \int_{\mathbb{R}^{2}} \ln (\|X-Y\|) \omega(Y, t) \mathrm{d} Y,
$$

where $\|\cdot\|$ is the Euclidean norm. On the other hand, considering a finite number $N$ of point vortices, then the viscosity vector can be written in the form $\omega(X, t)=$ 
$\sum_{i=1}^{N} k_{i} \delta\left(X-X_{i}(t)\right)$, where $\delta$ is the Dirac mass and where $k_{i}$ is the circulation of the ith-vortex. These two previous relations allow us to write the vortex dynamics as follows:

$$
\frac{\mathrm{d} x_{i}}{\mathrm{~d} t}=-\sum_{\substack{j=1 \\ j \neq i}}^{N} \frac{k_{j}}{2 \pi} \frac{y_{i}-y_{j}}{r_{i j}^{2}}, \quad \frac{\mathrm{d} y_{i}}{\mathrm{~d} t}=\sum_{\substack{j=1 \\ j \neq i}}^{N} \frac{k_{j}}{2 \pi} \frac{x_{i}-x_{j}}{r_{i j}^{2}},
$$

where $\left(x_{i}, y_{i}\right)$ is the position of the ith-vortex and where $r_{i j}^{2}:=\left(x_{i}-x_{j}\right)^{2}+\left(y_{i}-\right.$ $\left.y_{j}\right)^{2}$ is the square distance between the vortices $i$ and $j$.

As mentioned above, the aim of this paper is not to control the vortices but the displacement of particles. The idea is therefore to consider a particle (or passive tracer) as a point vortex with zero circulation and to apply a small amplitude control acting only on the passive tracer [16]. The control system is then written as follows: $\dot{q}=F_{0}(q)+u_{1} F_{1}(q)+u_{2} F_{2}(q)$ where $q:=\left(x, y, x_{1}, y_{1}, \cdots, x_{N}, y_{N}\right) \in \mathbb{R}^{2(1+N)}$ is the vector of positions of the particle and the vortices, where the control fields are $F_{1}(q)=\frac{\partial}{\partial x}$ and $F_{2}(q)=\frac{\partial}{\partial y}$, and where the drift $F_{0}$ is given by

$$
\begin{aligned}
F_{0}(q)= & -\sum_{j=1}^{N} \frac{k_{j}}{2 \pi} \frac{y-y_{j}}{r_{j}^{2}} \frac{\partial}{\partial x}+\sum_{j=1}^{N} \frac{k_{j}}{2 \pi} \frac{x-x_{j}}{r_{j}^{2}} \frac{\partial}{\partial y} \\
& +\sum_{i=1}^{N}\left(-\sum_{\substack{j=1 \\
j \neq i}}^{N} \frac{k_{j}}{2 \pi} \frac{y_{i}-y_{j}}{r_{i j}^{2}} \frac{\partial}{\partial x_{i}}+\sum_{\substack{j=1 \\
j \neq i}}^{N} \frac{k_{j}}{2 \pi} \frac{x_{i}-x_{j}}{r_{i j}^{2}} \frac{\partial}{\partial y_{i}}\right),
\end{aligned}
$$

with $r_{i}^{2}:=\left(x-x_{i}\right)^{2}+\left(y-y_{i}\right)^{2}, i=1, \cdots, N$. The optimal control problem of interest is then defined as follows: minimize the transfer energy $J(u):=\int_{0}^{T}\|u(t)\|^{2} \mathrm{~d} t$ to drive a passive particle from an initial point $\left(x_{0}, y_{0}\right) \in \mathbb{R}^{2}$ to a target point $\left(x_{f}, y_{f}\right) \in \mathbb{R}^{2}$, both given a priori, in a given finite time $T>0$. The initial positions of the vortices being also given.

\section{Case of one vortex}

\subsection{Pontryagin maximum principle and shooting function}

In the single vortex case one has $\left(\dot{x}_{1}, \dot{y}_{1}\right)=(0,0)$, that is the vortex is static and can be fixed to the origin of the reference frame. Hence, the control system may be reduced to

$$
\dot{q}(t)=F_{0}(q(t))+u_{1}(t) F_{1}(q(t))+u_{2}(t) F_{2}(q(t)),
$$

where $q=(x, y) \in \mathbb{R}^{2}$ (by a slight abuse of the notation since $\left(x_{1}, y_{1}\right)=(0,0)$ is constant) is the position of the particle and where the drift and the control fields are given by

$$
F_{0}(q)=\frac{\mu}{x^{2}+y^{2}}\left(-y \frac{\partial}{\partial x}+x \frac{\partial}{\partial y}\right), \quad F_{1}=\frac{\partial}{\partial x}, \quad F_{2}=\frac{\partial}{\partial y}, \quad \mu:=\frac{k}{2 \pi} .
$$


Explicitly the control problem in this case is written:

$$
\dot{x}(t)=-\frac{\mu y(t)}{x^{2}(t)+y^{2}(t)}+u_{1}(t), \quad \dot{y}(t)=\frac{\mu x(t)}{x^{2}(t)+y^{2}(t)}+u_{2}(t),
$$

Lemma 1. For any $q_{0}, q_{f}$ there exists a control joining $q_{0}$ to $q_{f}$ in time $T>0$.

Proof. Considering the polar coordinates $(r \cos \theta, r \sin \theta)=(x, y)$ and an adapted rotating frame for the control, $v=u e^{-i \theta}$, the control system (2) becomes $\dot{r}(t)=v_{1}(t), \dot{\theta}(t)=\mu / r(t)^{2}+v_{2}(t) / r(t)$. From $q_{0}$, we can apply a constant control $v(t)=\left(\alpha_{1}, 0\right)$ until the distance $\left\|q_{f}\right\|$ is reached and then apply a constant control $v(t)=\left(0, \alpha_{2}\right)$ until the target $x_{f}$ is reached, where $\alpha_{1}, \alpha_{2} \in \mathbb{R}$ are suitably chosen according to the time $T$.

Let $q_{0} \in \mathbb{R}^{2}$ denote the initial condition, $q_{f} \in \mathbb{R}^{2}$ the target and $T>0$ the transfer time. Let $u \in L^{\infty}\left([0, T], \mathbb{R}^{2}\right)$ be an optimal solution (assuming its existence) and let $q$ denote the associated optimal trajectory. According to the Pontryagin maximum principle [15], then there exists an absolutely continuous function $p:[0, T] \rightarrow \mathbb{R}^{2}$ satisfying the adjoint equation a.e. over $[0, T]$ :

$$
\dot{p}(t)=-\nabla_{q} H(q(t), p(t), u(t)),
$$

where $H(q, p, u):=p \cdot\left(F_{0}(q)+u_{1} F_{1}(q)+u_{2} F_{2}(q)\right)+p^{0}\|u\|^{2}$ is the pseudoHamiltonian. ${ }^{3}$ Besides, we have:

$$
p^{0} \leq 0, \text { the pair }\left(p, p^{0}\right) \text { never vanishes }
$$

and the optimal control satisfies the maximization condition a.e. over $[0, T]$ :

$$
H(q(t), p(t), u(t))=\max _{w \in \mathbb{R}^{2}} H(q(t), p(t), w) .
$$

Any quadruplet $\left(q, p, p^{0}, u\right)$ solution of (2) and (3)-(5) is called an extremal and is said to be abnormal if $p^{0}=0$ otherwise it is said to be normal. In the normal case, when $p^{0} \neq 0$, we can fix by homogeneity $p^{0}=-1 / 2$.

Definition 1. An extremal is a called BC-extremal if $q(0)=q_{0}$ and $q(T)=q_{f}$.

Proposition 1. Let $\left(q, p, p^{0}, u\right)$ be an extremal, then, the extremal is normal (that is there are no abnormal extremals) and the control $u$ is smooth.

Proof. Let us introduce the Hamiltonian lifts $H_{i}(z):=p \cdot F_{i}(q)$ with $z:=(q, p)$. If $p^{0}=0$, then the maximization condition leads to $H_{1}=H_{2}=0$, that is $p_{1}=p_{2}=0$, which contradicts the PMP. Hence $p^{0}<0$ and we can fix $p^{0}=$ $-1 / 2$. With this normalization, the control may be written in the feedback form $u(t)=\Phi(z(t))$ with $\Phi(z):=\left(H_{1}(z), H_{2}(z)\right)$ smooth. It is clear and well-known that in this case, the control law $t \mapsto u(t)$ is smooth.

\footnotetext{
3 The standard inner product is written $a \cdot b$, for $a, b$ in $\mathbb{R}^{2}$.
} 
Since we have only normal extremals we fix $p^{0}=-1 / 2$ and define the following true Hamiltonian (plugging $u$ in feedback form into the pseudo-Hamiltonian):

$$
\mathbf{H}(z):=H(z, \Phi(z))=H_{0}(z)+\frac{1}{2}\left(H_{1}^{2}(z)+H_{2}^{2}(z)\right)=H_{0}(z)+\frac{1}{2}\|p\|^{2} .
$$

Let's introduce the Hamiltonian system $\overrightarrow{\mathbf{H}}(z):=\left(\nabla_{p} \mathbf{H}(z),-\nabla_{q} \mathbf{H}(z)\right)$ and the pseudo-Hamiltonian system $\vec{H}(z, u):=\left(\nabla_{p} H(z, u),-\nabla_{q} H(z, u)\right)$. Then, we have:

Proposition 2. Let $(z, u)$ be an extremal $\left(p^{0}=-1 / 2\right.$ is implicit). Then:

$$
\dot{z}(t)=\vec{H}(z(t), u(t))=\overrightarrow{\mathbf{H}}(z(t))=\overrightarrow{H_{0}}(z(t))+(p(t), 0) .
$$

According to this proposition, we can define the exponential mapping:

$$
\exp _{t, q_{0}}\left(p_{0}\right):=\pi \circ \exp (t \overrightarrow{\mathbf{H}})\left(q_{0}, p_{0}\right),
$$

where $\pi(q, p):=q$ is the canonical projection on the state space and $\exp (t \overrightarrow{\mathbf{H}})\left(z_{0}\right)$ is the solution at time $t$ of $\dot{z}=\vec{H}(z)$ with the initial condition $z_{0}$. Finally, let us introduce the shooting function:

$$
\begin{aligned}
S: & \mathbb{R}^{2} \longrightarrow \mathbb{R}^{2} \\
& p_{0} \longmapsto S\left(p_{0}\right):=\exp _{T, q_{0}}\left(p_{0}\right)-q_{f} .
\end{aligned}
$$

Then, we have the classical following relation between BC-extremals and zeros of the shooting function.

Proposition 3. Let $(q, p, u)$ be a BC-extremal $\left(p^{0}=-1 / 2\right.$ is implicit $)$, then, $S(p(0))=0$. Conversely, let $p_{0} \in \mathbb{R}^{2}$ s.t. $S\left(p_{0}\right)=0$. Then, defining $z(t):=$ $\exp (t \overrightarrow{\mathbf{H}})\left(q_{0}, p_{0}\right)$ over $[0, T]$ and $u(t):=\Phi(z(t))$, the pair $(z, u)$ is a BC-extremal.

\subsection{Integration of the extremal solutions}

Writing the system in polar coordinates, the Hamiltonian becomes:

$$
\mathbf{H}\left(r, \theta, p_{r}, p_{\theta}\right)=p_{\theta} \frac{\mu}{r^{2}}+\frac{1}{2}\left(p_{r}^{2}+\frac{p_{\theta}^{2}}{r^{2}}\right) .
$$

Proposition 4. The system is Liouville integrable and, in polar coordinates, the extremals are given by: if $c:=p_{\theta}\left(2 \mu+p_{\theta}\right) \geq 0$, then

$$
\begin{aligned}
& r(t)=\sqrt{\frac{\left(c_{4} t+c_{5}\right)^{2}+c_{3}}{c_{4}}}, \quad p_{r}(t)=\frac{\left(c_{4} t+c_{5}\right)}{r(t)}, \\
& \theta(t)=\frac{c_{6}}{c_{4} \sqrt{c_{3}}}\left(\arctan \frac{c_{4} t+c_{5}}{\sqrt{c_{3}}}-\arctan \frac{c_{5}}{\sqrt{c_{3}}}\right),
\end{aligned}
$$

and if $c<0$, then

$$
\begin{aligned}
& r(t)=\sqrt{\frac{\left(c_{4} t+c_{5}\right)^{2}+c_{3}}{c_{4}}}, \quad p_{r}(t)=\frac{\left(c_{4} t+c_{5}\right)}{r(t)} \\
& \theta(t)=\frac{c_{6}}{c_{4} \sqrt{-c_{3}}}\left(\log \left|\frac{\left.c_{4} t+c_{5}\right)-\sqrt{-c_{3}}}{\left.c_{4} t+c_{5}\right)+\sqrt{-c_{3}}}\right|-\log \left|\frac{c_{5}-\sqrt{-c_{3}}}{c_{5}+\sqrt{-c_{3}}}\right|\right),
\end{aligned}
$$


with $c_{1}=p_{r}(0), c_{2}=p_{\theta}, c_{3}=c, c_{4}=c_{1}^{2}+\frac{c_{3}}{r_{0}^{2}}, c_{5}=r_{0}\left|c_{1}\right|, c_{6}=c_{4}\left(\mu+c_{2}\right)$.

Proof. The $\theta$ coordinate is cyclic, so $p_{\theta}$ defines a second first integral with $\mathbf{H}$. This guarantees the Liouville integrability of the system. For the integration of the extremals, one has to solve the following system:

$$
\dot{r}(t)=p_{r}(t), \quad \dot{p}_{r}(t)=\frac{2 \mu p_{\theta}}{r^{3}(t)}+\frac{p_{\theta}^{2}}{r^{3}(t)}, \quad \dot{\theta}(t)=\frac{\mu}{r^{2}(t)}+\frac{p_{\theta}}{r^{2}(t)}, \quad \dot{p_{\theta}}(t)=0,
$$

with $\left(r(0), \theta(0), p_{r}(0), p_{\theta}(0)\right)=:\left(r_{0}, \theta_{0}, c_{1}, c_{2}\right)$. Since $p_{\theta}=c_{2}$ is constant, we have from the two first equations that $\ddot{r}(t)=c_{3} / r^{3}(t)$, whence $\ddot{r}(t) \dot{r}(t)=$ $c_{3} \dot{r}(t) / r^{3}(t)$, with $c_{3}:=c_{2}\left(\mu+c_{2}\right)$. Solving this last equation we obtain $r(t)=$ $\sqrt{\left(\left(c_{4} t+c_{5}\right)^{2}+c_{3}\right) / c_{4}}$ with $c_{4}:=c_{1}^{2}+c_{3} / r_{0}^{2}$ and $c_{5}=r_{0}\left|c_{1}\right|$. One deduces $p_{r}(t)$ by differentiating this relation. The integration of $\theta$ depends on the sign of $c_{3}$. Indeed one has $\dot{\theta}(t)=c_{6} /\left(\left(c_{4} t+c_{5}\right)^{2}+c_{3}\right)$, with $c_{6}:=c_{4}\left(\mu+c_{2}\right)$, thus, if $c_{3} \geq 0$ one has the first case, otherwise one has the second.

\subsection{Numerical methods and results}

The HamPath ${ }^{4}$ code $[4,9]$ is used to compute the BC-extremals. A Newton-like algorithm is used to solve the shooting equation $S\left(p_{0}\right)=0$. Providing $\mathbf{H}$ and $S$ to HamPath, the code generates automatically the Jacobian of the shooting function. To make the implementation of $S$ easier, HamPath supplies the exponential mapping. Automatic Differentiation is used to produce $\overrightarrow{\mathbf{H}}$ and is combined with Runge-Kutta integrators to assemble the exponential mapping.

We present here, in the one vortex case, two examples. For the examples, we fix the initial condition to $q_{0}=(2,0)$, the transfer time to $T=10$ and we consider two targets: $q_{f}=(-2,0)$ and $q_{f}=(3,-3)$, to emphasize the influence of the vortex circulation (the strength of the drift being dependent to the distance between the particle and the vortex). The circulation is fixed to $k=2 \pi \mu$, with $\mu=2\left\|q_{0}\right\|$. The two results are detailed in the caption of Figure 1, where we represent the projection of the two BC-extremals in the state space, that is the trajectories, together with the control laws.

\section{Cases of 2,3 and 4 vortices}

\subsection{Pontryagin maximum principle and shooting function}

In the case of $N>1$ vortices, the vortices are not static and have to be considered in the dynamics. Let us recall that the control system has the form $\dot{q}=F_{0}(q)+$ $u_{1} F_{1}(q)+u_{2} F_{2}(q)$, where $F_{0}, F_{1}$, and $F_{2}$ are given in Section 2 , and that the state $q$ contains the position of the particle together with the positions of the $N$ vortices. In the control problem, see Section 2, the initial positions of the particle and the vortices are fixed. Let us denote by $q_{0} \in \mathbb{R}^{2(1+N)}$ the vector of these positions. On the other hand, the final position of the particle is fixed while the

\footnotetext{
${ }^{4}$ www.hampath.org
} 

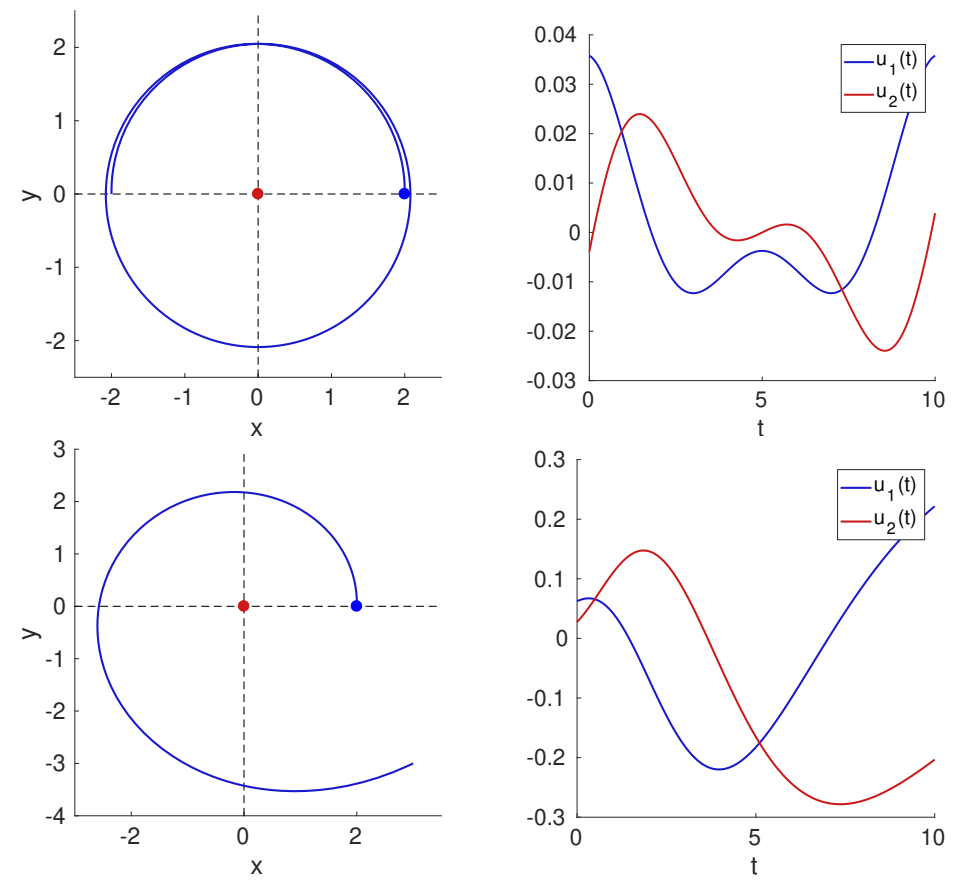

Fig. 1. One vortex. (Top) $q_{f}=(-2,0)$ and the cost is $J \approx 4.2675 \mathrm{e}-3$. (Bottom) $q_{f}=(3,-3)$ and the cost is $J \approx 5.5177 \mathrm{e}-1$. In both cases, the shooting equation $S=0$ is solved with a good accuracy of order 1e-12. On the left, we have the trajectory (in blue), the point vortex is represented by a red dot, the initial condition by a blue dot. The two control components are given on the right.

final positions of the $N$ vortices are free. Let us denote by $\left(x_{f}, y_{f}\right) \in \mathbb{R}^{2}$ the target for the particle. The transfer time is also fixed and denoted $T>0$. In order to apply the maximum principle, we define the pseudo-Hamiltonian:

$$
H(q, p, u):=p \cdot F_{0}(q)+u_{1} p \cdot F_{1}(q)+u_{2} p \cdot F_{2}(q)+p^{0}\left(u_{1}^{2}+u_{2}^{2}\right),
$$

where $p^{0}$ will be fixed to $-1 / 2$ according to:

Proposition 5. Let $\left(q, p, p^{0}, u\right)$ be a BC-extremal. Then, the extremal is normal and the control $u$ is smooth.

Proof. If $p^{0}=0$, then the maximization condition leads to $H_{1}=H_{2}=0$, that is $p_{x}=p_{y}=0$ all along the extremal. Decomposing the adjoint vector as $p:=$ $\left(p_{x}, p_{y}, p_{x_{1}}, p_{y_{1}}, \cdots, p_{x_{N}}, p_{y_{N}}\right)$, then, the transversality conditions implies that at the final time, $p_{x_{1}}(T), p_{y_{1}}(T), \ldots, p_{x_{N}}(T), p_{y_{N}}(T)$ are zero, which contradicts the PMP. Hence $p^{0}<0$ and we can fix $p^{0}=-1 / 2$. With this normalization, the control may be written in the feedback form $u(t)=\Phi(z(t))$ with $\Phi(z):=$ $\left(H_{1}(z), H_{2}(z)\right)$ smooth. Here again, the control law $t \mapsto u(t)$ is smooth. 
In the same spirit as in the one vortex case, one can define the following shooting function (with adapted and clear notation) to compute the BC-extremals:

$$
\begin{aligned}
S: \mathbb{R}^{2(1+N)} \longrightarrow & \mathbb{R}^{2(1+N)} \\
p_{0} \longmapsto & S\left(p_{0}\right):=\left(\begin{array}{rl}
x\left(T, q_{0}, p_{0}\right)-x_{f}, y\left(T, q_{0}, p_{0}\right)-y_{f}, \\
\left.p_{x_{1}}\left(T, q_{0}, p_{0}\right), \cdots, p_{y_{N}}\left(T, q_{0}, p_{0}\right)\right)
\end{array}\right.
\end{aligned}
$$

\subsection{Numerical results}

In the following examples, we fix the initial particle position to $\left(x_{0}, y_{0}\right)=(2,0)$ and the target to $\left(x_{f}, y_{f}\right)=(3,-3)$. To make a comparison with respect to the number of vortices we impose the condition $\Sigma_{i=1}^{N}\left|k_{i}\right|=k$, where $k$ is the circulation in the one vortex case, that is $k=2 \pi \mu$ with $\mu=2\left\|q_{0}\right\|$. The initial positions of the vortices are taken arbitrarily.

In general, the main difficulty to solve the shooting equations is the initialization of the adjoint vector due to the sensitivity of the underlying Newton method. In order to overcome this difficulty, we use the following algorithm:

- For two vortices, we first solve the subproblem where we set one of the circulation to zero, that is we solve a one vortex problem;

- Then, we use a path following algorithm [4] implemented in the HamPath software where the homotopic parameter is the value of the circulation which increase from 0 to the desired value;

- We repeat this procedure to obtain solutions for problems with more vortices.

We summarize in Table 1 the results in the one vortex case together with the new results for $N=2,3$ and 4 . The trajectories and the controls are given in Figures 2, 3 and 4, respectively for $N=2,3$ and 4 .

\begin{tabular}{|l|l|l|l|l|r|}
\hline Vortices & Circulations & Initial positions $\left(q_{0}\right)$ & Target & Cost & Figure \\
\hline \hline$N=1$ & $k=8 \pi$ & $(2,0)$ & $(-2,0)$ & 0.004 & 1 \\
\hline$N=1$ & $k=8 \pi$ & $(2,0)$ & $(3,-3)$ & 0.552 & 1 \\
\hline$N=2$ & $k_{1}=k_{2}=4 \pi$ & $(2,0,0,1,0,-1)$ & $(3,-3)$ & 1.947 & 2 \\
\hline$N=3$ & $k_{1}=k_{2}=k_{3}=8 \pi / 3$ & $(2,0,0,1,1,-1,-1,-1)$ & $(3,-3)$ & 0.406 & 3 \\
\hline$N=4$ & $k_{1}=k_{2}=k_{3}=k_{4}=2 \pi$ & $(2,0,1,0,0,1,-1,0,0,-1)$ & $(3,-3)$ & 0.335 & 4 \\
\hline
\end{tabular}

Table 1. In the two first columns are given the number of vortices with their circulations. In the third is given the initial positions of the particle and the vortices, the target for the particle being given in the fourth. The fifth column gives the cost associated to the computed BC-extremal, whose trajectory and control are plotted in the figure given by the last column.

\section{Conclusion}

In this article, we have solved the control problem in the cases $\mathrm{N}=1,2,3$ and 4 vortices. In the case of a single vortex, where it is trivial to show the integrability of the resulting Hamiltonian system, we have provided analytic expressions of 

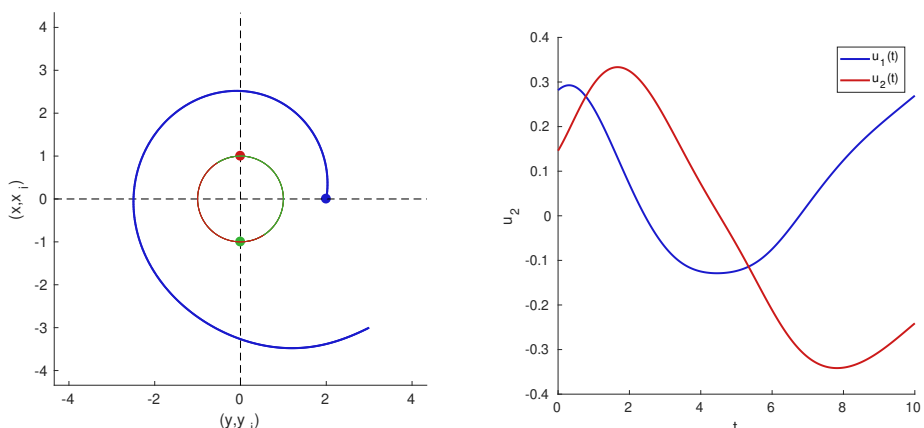

Fig. 2. Two vortices. Trajectory and control in the two vortices case. The initial adjoint vector being $p_{0}=(0.28,0.15,0.097,-0.47,-0.11,0.088)$ (see Table 1 for details).
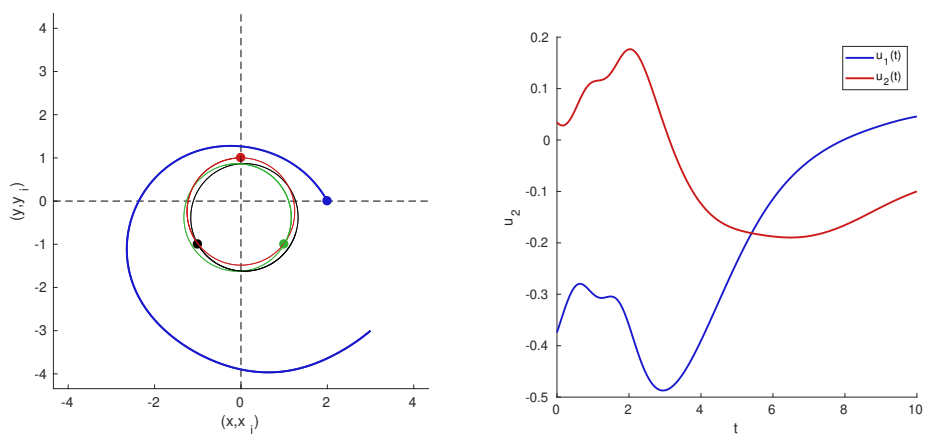

Fig. 3. Three vortices. Trajectory and control in the three vortices case. The initial adjoint vector being $p_{0}=(-0.37,-0.037,0.088,0.34,0.69,-0.48,-0.36,-0.026)$ (see Table 1 for details).
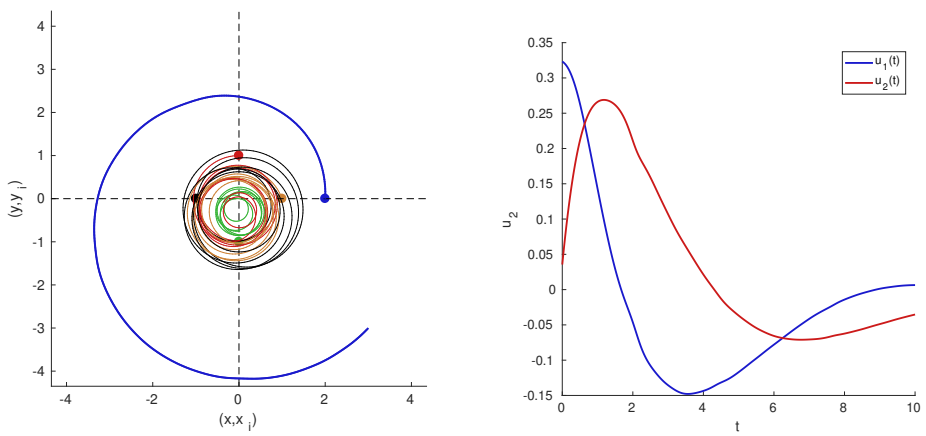

Fig. 4. Four vortices. Trajectory and control in the four vortices case. The initial adjoint vector is $p_{0}=(0.17,-0.0015,0.041,-0.18,-0.025,-0.26,0.11,0.11,-0.18,0.22)$ (see Table 1 for details).

extremals. In the other cases, we have limited ourselves to a numerical study of the problem by presenting some solutions obtained thanks to the HamPath software. Beyond 4 vortices, it is well known in the literature that the vortex 
system is chaotic (see for instance $[7,16]$ ), so controlling the particle in an environment containing more than 4 vortices no longer guarantees a solution to the problem. On the other hand, we have arbitrarily considered the initial positions of the vortices and we propose in future experiments to optimize this initial configuration in order to improve the results we have obtained.

\section{References}

1. A. Babiano, G. Boffetta, A. Provenzale and A. Vulpiani, Chaotic advection in point vortex models and two-dimensional turbulence, Physics of Fluids, 6 (1994), no. 7, pp. 2465-2474.

2. B. Bonnard, O. Cots and B. Wembe, A Zermelo navigation problem with a vortex singularity, 2019, hal-02296046v2.

3. B. Bonnard, L. Faubourg and E. Trélat, Mécanique céleste et contrôle des véhicules spatiaux, vol $\mathbf{5 1}$ of Mathématiques \& Applications, Springer-Verlag Berlin, 2006, 276 pages.

4. J.-B. Caillau, O. Cots and J. Gergaud, Differential continuation for regular optimal control problems, Optim. Methods Softw., 27 (2011), no. 2, pp. 177-196.

5. R. C. Calleja, E. J. Doedel \& C. García-Azpeitia, Choreographies in the n-vortex problem, Regul. Chaot. Dyn., 23 (2018), no. 5, pp. 595-612.

6. A. Chenciner, J. Gerver, R. Montgomery \& C. Simó, Simple Choreographic Motions of $N$ Bodies: A Preliminary Study, in Newton P., Holmes P., Weinstein A. (eds) Geometry, Mechanics, and Dynamics. Springer, New York, NY, 2002, pp. 287-308.

7. Y. Chen and T. Kolokolnikov, Collective behaviour of large number of vortex in the plane, Proc. R. Soc, A, 469 (2013), no. 2156, 12 pages.

8. R. Chertovskih and D. Karamzin and N.T. Khalil and F. Lobo Pereira Regular path-constrained time-optimal control problems in three-dimensional flow fields, European Journal of Control. https://doi.org/10.1016/j.ejcon.2020.02.003,

9. O. Cots, Contrôle optimal géométrique : méthodes homotopiques et applications, Phd thesis, Institut Mathématiques de Bourgogne, Dijon, France, 2012.

10. J. Liu and H. Hu Biological Inspiration: From Carangiform Fish to Multi-Joint Robotic Fish. Springer Nature, J. Bionic Eng., 7 (2010), no. 1, pp. 35-48.

11. K. Meyer, G. Hall \& D. C. Offin, Introduction to Hamiltonian Dynamical Systems and the N-Body Problem, vol. 90 of Applied Mathematical Sciences, Springer-Verlag New York, 2009, 399 pages.

12. P. K. Newton, The N-Vortex Problem: Analytical Techniques, Springer Science \& Business Media, vol. 145 of Applied Mathematical Sciences, 2013, 420 pages.

13. F. L. Pereira and, T. Grilo and S. Gama, Optimal Multi-process Control of a Two Vortex Driven Particle in the Plane, IFAC-PapersOnLine, 50 (2017), no. 1, pp. 2193-2198.

14. H. Poincaré, Euvres, Gauthier-Villars, 1952.

15. L. S. Pontryagin, V. G. Boltyanskiı̌, R. V. Gamkrelidze and E. F. Mishchenko, The Mathematical Theory of Optimal Processes, Translated from the Russian by K. N. Trirogoff, edited by L. W. Neustadt, Interscience Publishers John Wiley \& Sons, Inc., New York-London (1962).

16. B. Protas, Vortex dynamics models in flow control problems, Nonlinearity of IOP Science, 21 (2008), no.9, pp. R203-R250.

17. D. Vainchtein, I. Mezi, Vortex-based control algorithms, Control of fluid flow, Berlin: Springer (2006), 189-212. 